\title{
'Hard-Core' Diels-Alder
}

\section{Key words}

diamond nanoparticles

Diels-Alder reaction

electrophilic aromatic substitution

\section{SYNFACTDin}

Significance: A Diels-Alder reaction is used for the first time to functionalize the surface of diamond nanoparticles. Direct $\mathrm{C}-\mathrm{C}$ coupling is achieved by reacting $\mathrm{o}$-quinodimethanes with $\pi$-bonds on the surface of thermally annealed nanodiamond. Increasing the annealing temperature and introducing electron-withdrawing groups to the diene both lead to higher surface loading.
Comment: This method offers facile modification of surface properties of nanodiamond. The arylated particles are very stable and can be further decorated by electrophilic aromatic substitution. For instance, 4-carboxy-o-quinodimethane-functionalized particles $\mathbf{2 b}$ are soluble in water and PBS buffer, while Oregon Green tagged conjugate 5 can be purified by conventional column chromatography. 